Journal of Applied AnALysis

Vol. 13, No. 2 (2007), pp. 291-302

\title{
GENERALIZED VECTOR VARIATIONAL INEQUALITIES IN TOPOLOGICAL VECTOR SPACES
}

\author{
T. JABAROOTIAN and J. ZAFARANI ${ }^{1}$ \\ Received december 11, 2006 and, in revised form, May 9, 2007
}

\begin{abstract}
The solvability of the generalized weak vector implicit variational inequality problem, generalized strong vector implicit variational inequality problem and generalized vector variational inequality problem are proved by using a generalized Fan's KKM theorem. Our results extend and unify corresponding results of other authors.
\end{abstract}

\section{INTRODUCTION AND PRELIMINARIES}

The vector variational inequality (for short, VVI) was first introduced by Giannessi [8] in the setting of finite dimensional Euclidean space. Throughout over twenty six years, existence theorems of solution of vector variational inequalities in various situations have been studied by many authors (see for example, [1]-[4], [8]-[12], [14]-[16], [18], [21]-[23]). At the same time, vector variational inequality has found many of its applications in vector

2000 Mathematics Subject Classification. 47J20, 49J40.

Key words and phrases. Fan's KKM theorem, vector variational inequality, strong vector variational inequality, strong vector implicit variational inequality.

${ }^{1}$ The second author was partially supported by the Center of Excellence for Mathematics (University of Isfahan).

ISSN 1425-6908（C) Heldermann Verlag. 
optimization [21], approximate vector optimization [1], vector equilibria [9], [12] and vector traffic equilibria [10], [23].

Assume $X$ and $Y$ are real Hausdorff topological vector spaces, $L(X, Y)$ is the space of all continuous linear operators from $X$ into $Y$. When $Y$ is the set $\mathbb{R}$ of real numbers, $L(X, Y)$ is the usual dual space $X^{*}$ of $X$. For any $x \in X$ and any $u \in L(X, Y)$, we shall write the value $u(x)$ as $\langle u, x\rangle$. Let $\sigma$ be a family of all bounded subsets of $X$ whose union is total in $X$, i.e., the linear hull of $\cup\{S: S \in \sigma\}$ is dense in $X$. Let $\mathfrak{B}$ be a neighborhood base of 0 in $Y$. When $S$ runs through $\sigma, V$ through $\mathfrak{B}$, the family

$$
M(S, V)=\left\{u \in L(X, Y): \bigcup_{x \in S}\langle u, x\rangle \subset V\right\}
$$

is a neighborhood base of 0 in $L(X, Y)$ for a unique translation-invariant topology, called the topology of uniform convergence on the sets $S \in \sigma$, or, briefly the $\sigma$-topology [17]. Suppose that $K$ is a nonempty convex subset of $X, T: K \rightarrow 2^{L(X, Y)}$ a set valued mapping, $g: K \rightarrow K$ and $\{D(x): x \in$ $K\}$ is a family of convex cones in $Y$. In [16], Rapcsák introduced the week order in Banach spaces. Based on this new order, he introduced a vector variational inequality and three vector complementarity problems and discussed some relations between them. Huang and Li in [13] continued this kind of problems and answered an open question posed by Rapcsák concerning the existence of a solution to vector variational inequality and weak vector complementarity problems. In this article, we consider the following problems:

(I) Generalized weak vector implicit variational inequality (for short, GWVIVI): find $x_{0} \in K$ such that for each $y \in K$,

$$
\left\langle T\left(x_{0}\right), y-g\left(x_{0}\right)\right\rangle \cap-D\left(x_{0}\right)=\emptyset .
$$

(II) Generalized strong vector implicit variational inequality (for short, GSVIVI): find $x_{0} \in K$ such that for each $y \in K$,

$$
\left\langle T\left(x_{0}\right), y-g\left(x_{0}\right)\right\rangle \subset \operatorname{cl} D\left(x_{0}\right) .
$$

(III) Generalized vector variational inequality (for short, GVVI): find $x_{0} \in$ $K$ such that for each $y \in K$,

$$
\left\langle T\left(x_{0}\right), y-x_{0}\right\rangle \nsubseteq-\mathrm{cl} D\left(x_{0}\right) \backslash\{0\},
$$

where $\left\langle T\left(x_{0}\right), y-x_{0}\right\rangle=\bigcup_{u \in T\left(x_{0}\right)}\left\langle u, y-x_{0}\right\rangle$.

When $g$ is the identity mapping on $K$, then GWVIVI reduces to generalized weak vector variational inequality (for short, GWVVI) and GSVIVI reduces to Generalized strong vector variational inequality (for short, GSVVI). If $T$ is single-valued, then GWVIVI reduces to weak vector implicit variational inequality (for short, WVIVI) which has been studied by Huang and Li [13]. Moreover, when $g$ is also the identity mapping on $K$, then GWVIVI reduces 
to vector variational inequality (for short, VVI) which has been studied by Rapcsák [16]. If $T$ is single-valued, then GVVI reduces to strong vector variational inequality (for short, SVVI) which has been studied by Fang and Huang [7].

Let $D$ be a convex cone. In [16], Rapcsák introduced the following order:

$$
y \leq_{D} x \Leftrightarrow x-y \in D,
$$

and

$$
y \not \leq D x \Leftrightarrow x-y \notin D .
$$

Since $\operatorname{cl} D$ is a closed convex cone, where $\operatorname{cl} D$ denotes the closure of $D$, then

$$
y \leq_{\mathrm{cl} D} x \Leftrightarrow x-y \in \operatorname{cl} D, \quad \forall x, y \in Y,
$$

and

$$
y \not \mathbb{c l}_{\mathrm{cl}} x \Leftrightarrow x-y \notin \mathrm{cl} D, \quad \forall x, y \in Y .
$$

The above order satisfies the following properties:

$$
\begin{aligned}
& y \not \mathbb{D}_{D} x \Leftrightarrow y+w \not \mathbb{D}_{D} x+w \quad \forall x, y, w \in Y, \\
& y \not \leq D x \Leftrightarrow \lambda y \not \leq D \lambda x \quad \forall x, y \in Y \text { and } \lambda>0 .
\end{aligned}
$$

We say that a convex cone $D$ is acute, if $\operatorname{cl} D$ is pointed.

Lemma 1.1 ([16]). Let $D$ be a convex cone in $Y$ and $x, y \in Y$. Then

(i) $0 \varliminf_{D} y$ and $x \leq_{D} y$ implies that $0 \varliminf_{D} x$.

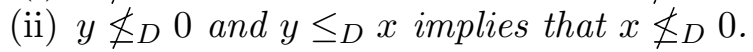

(iii) If moreover, $D$ is acute and $0 \notin-D, 0 \leq_{\operatorname{cl} D}$ y implies that $y \not{ }_{D} 0$.

Throughout this paper, we always use the above order due to Rapcsák [16].

Let $X$ be a nonempty set, we shall denote by $2^{X}$ the family of all subsets of $X$, by $\mathcal{F}(X)$ the family of all nonempty finite subsets of $X$. Let $Y$ be a nonempty set, $X$ a topological space and $F: Y \rightarrow 2^{X}$ a set-valued mapping. Then $F$ is said to be transfer closed-valued if for any $(y, x) \in Y \times X$ with $x \notin F(y)$, there exists $y^{\prime} \in Y$ such that $x \notin \mathrm{cl} F\left(y^{\prime}\right)$. One can show that [19], this definition is equivalent to saying that

$$
\bigcap_{y \in Y} F(y)=\bigcap_{y \in Y} \operatorname{cl} F(y) .
$$

If $B \subseteq Y$ and $A \subseteq X$, then we call $F: B \rightarrow 2^{A}$ transfer closed-valued if the multi-valued mapping $y \rightarrow F(y) \bigcap A$ is transfer closed-valued. In the case where $X=Y$ and $A=B$, we call $F$ transfer closed-valued on $A$. Let $K$ be a convex subset of a vector space $X$. Then a mapping $F: K \rightarrow 2^{X}$ is called a KKM mapping if for each nonempty finite subset $A$ of $K$, co $A \subset F(A)$, where co $A$ denotes the convex hull of $A$, and $F(A)=\bigcup\{F(x): x \in A\}$. 
Theorem 1.1 ([5]). Let $K$ be a nonempty convex subset of a Hausdorff t.v.s. $X$. Suppose that $\Gamma, \hat{\Gamma}: K \rightarrow 2^{K}$ are two set-valued mappings such that following conditions are satisfied:

(A1) $\hat{\Gamma}(x) \subseteq \Gamma(x)$ for all $x \in K$,

(A2) $\hat{\Gamma}$ is a KKM map,

(A3) for each $A \in \mathcal{F}(K), \Gamma$ is transfer closed-valued on co $A$,

(A4) for each $A \in \mathcal{F}(K), \operatorname{cl}_{K}\left(\bigcap_{x \in \operatorname{co} A} \Gamma(x)\right) \cap \operatorname{co} A=\left(\bigcap_{x \in \operatorname{co} A} \Gamma(x)\right) \cap \operatorname{co} A$,

(A5) there is a nonempty compact convex set $B \subseteq K$ such that $\operatorname{cl}_{K}\left(\bigcap_{x \in B} \Gamma(x)\right)$ is compact.

Then, $\bigcap_{x \in K} \Gamma(x) \neq \emptyset$.

Definition 1.1. Let $X$ and $Y$ be two topological spaces and $T: X \rightarrow 2^{Y}$ be a set-valued mapping. Then

(1) $T$ is said to be lower semi continuous (l.s.c.), iff for each open set $B \subset Y, T^{-}(B)=\{x \in X: T(x) \cap B \neq \varnothing\}$ is open in $X$.

(2) $T$ is said to be closed (resp. open), iff the set $G_{r}(T)=\{(x, y) \in$ $X \times Y: y \in T(x)\}$ is closed (resp. open) in $X \times Y$.

Lemma $1.2([20])$. Let $X$ and $Y$ be two topological spaces and $T: X \rightarrow 2^{Y}$ be a set-valued mapping. Then $T$ is l.s.c. if and only if for any $y \in T(x)$ and any net $\left\{x_{\alpha}\right\}$ in $X$ that converges to $x$, there exists a net $\left\{y_{\alpha}\right\}$ such that $y_{\alpha} \in T\left(x_{\alpha}\right)$ with $y_{\alpha} \rightarrow y$.

\section{MAIN RESUltS}

In this section, using Theorem 1.1, the solvability of GWVIVI, GSVIVI and GVVI are proved. In the following result, we obtain a solution for GSVIVI.

Theorem 2.1. Assume that the following conditions are satisfied:

(i) For each finite subset $A$ of $K$, the set-valued mapping $\Gamma: K \rightarrow 2^{K}$ defined by

$$
\Gamma(y)=\{x \in K:\langle T(x), y-g(x)\rangle \subseteq \operatorname{cl} D(x)\}
$$

is transfer closed valued on $\operatorname{co}(A)$.

(ii) For each finite subset $A$ of $K, x, y \in \operatorname{co}(A)$ and for each net $\left\{x_{\alpha}\right\}$ in $K$ converging to $x$, if $x_{\alpha} \in \Gamma(z)$ for all $z \in \operatorname{co}(A)$ and for all $\alpha$, then $x \in \Gamma(y)$.

(iii) For any fixed $x \in K$, the set $\{y \in K:\langle T(x), y-g(x)\rangle \nsubseteq \operatorname{cl} D(x)\}$ is convex.

(iv) For each $x \in K$, we have $\langle T(x), x-g(x)\rangle \subseteq \operatorname{cl} D(x)$. 
(v) There is a nonempty compact set $M \subset K$, and there is a nonempty compact convex set $B \subset K$ such that for each $x \in K \backslash M$, there exists $y \in B$ such that $x \notin \Gamma(y)$.

Then GSVIVI has a solution.

Proof. We show that $\Gamma$ is a KKM mapping. Since if $\Gamma$ is not a KKM mapping, then there exists $\left\{x_{1}, x_{2}, \ldots, x_{n}\right\} \subset K, t_{i} \geq 0, i=1,2, \ldots, n$ with $\sum_{i=1}^{n} t_{i}=1$ such that $x=\sum_{i=1}^{n} t_{i} x_{i} \notin \bigcup_{i=1}^{n} \Gamma\left(x_{i}\right)$. Thus for any $i=1,2, \ldots, n$, we have

$$
\left\langle T(x), x_{i}-g(x)\right\rangle \nsubseteq \mathrm{cl} D(x) .
$$

From conditions (iii), we deduce that $\langle T(x), x-g(x)\rangle \nsubseteq \mathrm{cl} D(x)$, which contradicts condition (iv). Hence, $\Gamma$ is a KKM mapping. Thus all of the conditions of Theorem 1.1 are fulfilled by the mapping $\hat{\Gamma}=\Gamma$. Therefore,

$$
\bigcap_{y \in K} \Gamma(y) \neq \emptyset \text {. }
$$

Consequently, there is $x_{0} \in K$ such that $\left\langle T\left(x_{0}\right), y-g\left(x_{0}\right)\right\rangle \subseteq \operatorname{cl} D\left(x_{0}\right)$ for all $y \in K$.

Remark 2.1. Theorem 2.1 improves Theorem 3.2 in [13]. In Theorem 2.1, we can replace conditions (iii) and (iv), by the following condition:

(iii) ${ }^{\prime} T$ is properly quasimonotone with respect to $g$, i.e. for all $n \in \mathbb{N}$ and for all vectors $x_{1}, x_{2}, \ldots, x_{n} \in K$, and scalars $t_{i}>0, i=1,2, \ldots, n$ with $\sum_{i=1}^{n} t_{i}=1$ and $x=\sum_{i=1}^{n} t_{i} x_{i},\left\langle T(x), x_{i}-g(x)\right\rangle \subseteq \operatorname{cl} D(x)$ holds for some $i$.

In fact, by a similar proof, we can show that $\Gamma$ is a KKM mapping. Thus we conclude an improvement of Theorem 2.1 in [6].

From Lemma 1.1 and by an argument similar to the proof of the part (i) of Theorem 3.1 in [13], we have the following result in our case.

Theorem 2.2. Let $D(x)$ be an acute convex cone and $0 \notin-D(x)$ for each $x \in K$. Then if $x_{0}$ solves GSVIVI, $x_{0}$ solves also GWVIVI.

In the above result, when, $g$ is the identity mapping on $K$, if $x_{0}$ solves GSVVI, then $x_{0}$ solves also GWVVI. Hence, we improve Theorem 3.3 in [13].

When $\Gamma(y)$ is closed with respect to $K$ for each $y \in K$, then $\Gamma$ satisfies conditions (i) and (ii). In the following result we establish the closedness of $\Gamma$. 
Corollary 2.1. Let $X$ be metrizable. Assume that the following conditions are satisfied:

(i) The set-valued mapping $W: K \rightarrow 2^{Y}$ is defined by $W(x)=Y \backslash$ $(\mathrm{cl} D(x))$ is open.

(ii) $T: K \rightarrow 2^{L(X, Y)}$ is l.s.c.

(iii) $g$ is continuous.

(iv) For each $x \in K$, we have $\langle T(x), x-g(x)\rangle \subseteq \mathrm{cl} D(x)$ and the set $\{y \in K:\langle T(x), y-g(x)\rangle \nsubseteq \mathrm{cl} D(x)\}$ is convex.

(v) There is a nonempty compact set $M \subset K$, and there is a nonempty compact convex set $B \subset K$ such that for each $x \in K \backslash M$, there exists $y \in B$ such that

$$
\langle T(x), y-g(x)\rangle \nsubseteq \mathrm{cl} D(x) .
$$

Then GSVIVI has a solution.

Proof. By Theorem 2.1, it is enough to show that for each $y \in K$, the set

$$
\Gamma(y)=\{x \in K:\langle T(x), y-g(x)\rangle \subseteq \operatorname{cl} D(x)\}
$$

is closed with respect to $K$. Let $\left\{x_{n}\right\}$ be a sequence in $\Gamma(y)$ convergent to $\bar{x} \in K$. By lower seimcontinuity of $T$ and Lemma 1.2, for all $v \in T(\bar{x})$, there exists a sequence $v_{n} \in T\left(x_{n}\right)$ converging to $v$. Set $z_{n}=\left\langle v_{n}, y-g\left(x_{n}\right)\right\rangle$, then since $x_{n} \in \Gamma(y)$, we have $z_{n} \in \operatorname{cl} D\left(x_{n}\right)$. Therefore, $\left(x_{n}, z_{n}\right) \notin G_{r}(W)$. By continuity of $g, y-g\left(x_{n}\right)$ is a convergent sequence and hence it is bounded. Thus, Proposition 2.3 of [3] implies that $z=\lim _{n} z_{n}=\langle v, y-g(\bar{x})\rangle$. Since $G_{r}(W)$ is open, then $(\bar{x}, z) \notin G_{r}(W)$, hence, $\bar{x} \in \Gamma(y)$, and the proof is complete.

Remark 2.2. Corollary 2.1 improves Theorem 3.2 of [13] in many aspects. Corollary 2.1 also improves Theorem 3.3 of [13] for existence theorem of GWVIVI by using Theorem 2.2.

When $K$ is a compact subset of $X$, then we can omit the condition of metrizability of $X$. Hence we can deduce from Theorem 2.1 the following result.

Corollary 2.2. Let $K$ be a nonempty compact convex subset of $X$. Assume that the conditions (i)-(iv) of the Corollary 2.1 are satisfied. Then GSVIVI has a solution.

We will establish now an existence result for GVVI without any monotonicity. 
Theorem 2.3. Assume that $\{D(x): x \in K\}$ is a family of acute convex cones in $Y$ and the following conditions are satisfied:

(i) For each finite subset $A$ of $K$, the set-valued mapping $\Gamma: K \rightarrow 2^{K}$ defined by

$$
\Gamma(y)=\{x \in K:\langle T(x), y-x\rangle \nsubseteq-\operatorname{cl} D(x) \backslash\{0\}\}
$$

is transfer closed valued on $\operatorname{co}(A)$.

(ii) For each finite subset $A$ of $K, x, y \in \operatorname{co}(A)$ and for each net $\left\{x_{\alpha}\right\}$ in $K$ converging to $x$, if $x_{\alpha} \in \Gamma(z)$ for all $z \in \operatorname{co}(A)$ and for all $\alpha$, then $x \in \Gamma(y)$.

(iii) There is a nonempty compact set $M \subset K$, and there is a nonempty compact convex set $B \subset K$ such that for each $x \in K \backslash M$, there exists $y \in B$ such that $x \notin \Gamma(y)$.

Then GVVI has a solution.

Proof. We show that $\Gamma$ is a KKM mapping. Since if $\Gamma$ is not a KKM mapping, then there exists $\left\{x_{1}, x_{2}, \ldots, x_{n}\right\} \subset K, t_{i} \geq 0, i=1,2, \ldots, n$ with $\sum_{i=1}^{n} t_{i}=1$ such that $x=\sum_{i=1}^{n} t_{i} x_{i} \notin \bigcup_{i=1}^{n} \Gamma\left(x_{i}\right)$. Thus for any $i=1,2, \ldots, n$ and any $u \in T(x)$, we have

$$
\left\langle u, x_{i}-x\right\rangle \in-\operatorname{cl} D(x) \backslash\{0\} .
$$

Since $\operatorname{cl} D(x)$ is pointed convex cone, therefore $-\operatorname{cl} D(x) \backslash\{0\}$ is convex and we obtain

$$
\sum_{i=1}^{n} t_{i}\left\langle u, x_{i}-x\right\rangle=\left\langle u, \sum_{i=1}^{n} t_{i} x_{i}-x\right\rangle=\langle u, x-x\rangle=0 \in-\operatorname{cl} D(x) \backslash\{0\},
$$

which is a contradiction. Hence, $\Gamma$ is a KKM mapping. Thus all of the conditions of Theorem 1.1 are fulfilled by the mapping $\hat{\Gamma}=\Gamma$. Therefore,

$$
\bigcap_{y \in K} \Gamma(y) \neq \emptyset \text {. }
$$

Hence, there exists $x_{0} \in \bigcap_{y \in K} \Gamma(y)$ which is a solution for GVVI.

As a consequence of Theorem 2.3, we obtain the following result which generalizes Theorem 2.1 of [7].

Corollary 2.3. Assume that $\{D(x): x \in K\}$ is a family of acute convex cones in $Y$ and the following conditions are satisfied:

(i) For each $y \in K$,

$$
\Gamma(y)=\{x \in K:\langle T(x), y-x\rangle \nsubseteq-\operatorname{cl} D(x) \backslash\{0\}\}
$$

is closed. 
(ii) There is a nonempty compact set $M \subset K$, and there is a nonempty compact convex set $B \subset K$ such that for each $x \in K \backslash M$, there exists $y \in B$ such that $x \notin \Gamma(y)$.

Then GVVI has a solution.

Theorems 2.1-2.3 and their corollaries present the solvability of GSVIVI, GVVI and GWVIVI without monotonicity. In the following we prove the solvability of a GVVI under suitable generalized monotonicity. First we give some concepts and a lemma.

Definition 2.1. A set-valued mapping $T: K \rightarrow 2^{L(X, Y)}$ is said to be strongly pseudomonotone iff for each $x, y \in K$,

$$
\langle T(x), y-x)\rangle \nsubseteq-\operatorname{cl} D(x) \backslash\{0\} \Rightarrow\langle T(y), y-x\rangle \subseteq \operatorname{cl} D(x) .
$$

The above definition of pseudomontonicity is stronger than the one in common sense, see Remark 2.2 and Example 2.2 in [7].

Definition 2.2. A set-valued mapping $T: K \rightarrow 2^{L(X, Y)}$ is said to be lower hemicontinuous (for short, l.h.c.) on $K$ iff for every $x, y \in K$ the set-valued mapping

$$
t \in[0,1] \mapsto\langle T(x+t(y-x)), y-x\rangle,
$$

is l.s.c. at $0^{+}$, where

$$
\langle T(x+t(y-x)), y-x\rangle=\{\langle v, y-x\rangle: v \in T(x+t(y-x))\} .
$$

We show that under some restrictions on our mappings, we obtain the equivalence between existence of solution for GVVI and the following problem:

(IV) Find $x_{0} \in K$ such that for each $y \in K$,

$$
\left\langle T(y), y-x_{0}\right\rangle \subseteq \operatorname{cl} D\left(x_{0}\right) .
$$

Lemma 2.1. Let $T: K \rightarrow 2^{L(X, Y)}$ be l.h.c., strongly pseudomonotone mapping and $\{D(x): x \in K\}$ be a family of acute convex cones in $Y$. Then, solutions of problem (IV) and GVVI coincide.

Proof. Suppose that $x_{0} \in K$ is a solution of GVVI, then for each $y \in K$,

$$
\left\langle T\left(x_{0}\right), y-x_{0}\right\rangle \nsubseteq-\operatorname{cl} D\left(x_{0}\right) \backslash\{0\} .
$$

Since $T$ is strongly pseudomonotone, we deduce that

$$
\left\langle T(y), y-x_{0}\right\rangle \subseteq \operatorname{cl} D\left(x_{0}\right) .
$$

Conversely, suppose that we can find $x_{0} \in K$ such that for each $y \in K$,

$$
\left\langle T(y), y-x_{0}\right\rangle \subseteq \operatorname{cl} D\left(x_{0}\right) .
$$


Then by substituting $x_{t}=x_{0}+t\left(y-x_{0}\right)$ for $t \in[0,1]$, into (2.1), we have

$$
\left.t\left\langle T\left(x_{t}\right), y-x_{0}\right)\right\rangle \subseteq \operatorname{cl} D\left(x_{0}\right) .
$$

Since $\operatorname{cl} D\left(x_{0}\right)$ is a cone,

$$
\left\langle T\left(x_{t}\right), y-x_{0}\right\rangle \subseteq \operatorname{cl} D\left(x_{0}\right) .
$$

Suppose in the contrary

$$
\left\langle T\left(x_{0}\right), y-x_{0}\right\rangle \cap\left(Y \backslash \operatorname{cl} D\left(x_{0}\right)\right) \neq \emptyset .
$$

As $T$ is l.h.c., we have

$$
\left\langle T\left(x_{t}\right), y-x_{0}\right\rangle \cap\left(Y \backslash \operatorname{cl} D\left(x_{0}\right)\right) \neq \emptyset,
$$

for sufficiently small $t$, which contradicts (2.2). Hence,

$$
\left\langle T\left(x_{0}\right), y-x_{0}\right\rangle \subseteq \operatorname{cl} D\left(x_{0}\right),
$$

which implies that

$$
\left\langle T\left(x_{0}\right), y-x_{0}\right\rangle \nsubseteq-\operatorname{cl} D\left(x_{0}\right) \backslash\{0\} .
$$

Remark 2.3. Lemma 2.1 is a vector version of Lemma 2.2 of [7].

Theorem 2.4. Let $\{D(x): x \in K\}$ be a family of acute convex cones in $Y$. Assume that the following conditions are satisfied.

(i) For each finite subset $A$ of $K$, the set-valued mapping $\Gamma: K \rightarrow 2^{K}$ defined by

$$
\Gamma(y)=\{x \in K:\langle T(y), y-x\rangle \subseteq \operatorname{cl} D(x)\}
$$

is transfer closed valued on $\operatorname{co}(A)$.

(ii) For each finite subset $A$ of $K, x, y \in \operatorname{co}(A)$ and for each net $\left\{x_{\alpha}\right\}$ in $K$ converging to $x$, if $x_{\alpha} \in \Gamma(z)$ for all $z \in \operatorname{co}(A)$ and for all $\alpha$, then $x \in \Gamma(y)$.

(iii) $T: K \rightarrow 2^{L(X, Y)}$ be a l.h.c. and strongly pseudomonotone mapping.

(iv) There is a nonempty compact set $M \subset K$, and there is a nonempty compact convex set $B \subset K$ such that for each $x \in K \backslash M$, there exists $y \in B$ such that $x \notin \Gamma(y)$.

Then GVVI has a solution.

Proof. For each $y \in K$, we define the set-valued mapping $\hat{\Gamma}: K \rightarrow 2^{K}$ by

$$
\hat{\Gamma}(y):=\{x \in K:\langle T(x), y-x\rangle \nsubseteq-\operatorname{cl} D(x) \backslash\{0\}\} .
$$

By a similar argument as that in the proof of Theorem 2.3, one can show that $\hat{\Gamma}$ is a KKM mapping. From definition of strongly pseudomonotonicity, 
we have $\hat{\Gamma}(y) \subseteq \Gamma(y)$ for all $y \in K$. Thus all of the conditions of Theorem 1.1 are fulfilled by the mappings $\hat{\Gamma}$ and $\Gamma$. Therefore,

$$
\bigcap_{y \in K} \Gamma(y) \neq \emptyset \text {. }
$$

Hence Problem (IV) has a solution and from Lemma 2.1 we deduce that GVVI has a solution.

Remark 2.4. In Theorem 2.4, if the set

$$
\Gamma(y)=\{x \in K:\langle T(y), y-x\rangle \subseteq \operatorname{cl} D(x)\}
$$

is closed for any $y \in K$, then conditions (i) and (ii) hold. In the following result, we will establish the closedness of $\Gamma(y)$ for each $y \in K$.

Corollary 2.4. Assume that $\{D(x): x \in K\}$ is a family of acute convex cones in $Y$ and the following conditions are satisfied:

(i) The set-valued mapping cl $D: K \rightarrow 2^{Y}$ is closed.

(ii) $T: K \rightarrow 2^{L(X, Y)}$ is strongly pseudomonotone and l.h.c.

(iii) There is a nonempty compact set $M \subset K$, and there is a nonempty compact convex set $B \subset K$ such that for each $x \in K \backslash M$, there exists $y \in B$ such that $x \notin \Gamma(y)$.

Then GVVI has a solution.

Proof. By Theorem 2.4, it is enough to show that for each $y \in K$, the set

$$
\Gamma(y)=\{x \in K:\langle T(y), y-x\rangle \subseteq \operatorname{cl} D(x)\}
$$

is closed. Let $\left\{x_{\alpha}\right\}$ be a net in $\Gamma(y)$ convergent to $x_{0} \in K$. Since $x_{\alpha} \in \Gamma(y)$, for each $v \in T(y)$, we have

$$
z_{\alpha}=\left\langle v, y-x_{\alpha}\right\rangle \in \operatorname{cl} D\left(x_{\alpha}\right)
$$

then $\left(x_{\alpha}, z_{\alpha}\right) \in G_{r}(\operatorname{cl} D)$. Moreover, we have

$$
z_{0}=\lim _{\alpha} z_{\alpha}=\left\langle v, y-x_{0}\right\rangle
$$

Since $G_{r}(\operatorname{cl} D)$ is closed, then $\left(x_{0}, z_{0}\right) \in G_{r}(\operatorname{cl} D)$ and hence,

$$
\left\langle v, y-x_{0}\right\rangle \in \operatorname{cl} D\left(x_{0}\right) .
$$

Thus, $x_{0} \in \Gamma(y)$, this means $\Gamma(y)$ is a closed subset of $K$.

Remark 2.5. Corollary 2.4 improves Theorem 2.3 of [7]. 
Acknowledgment. The authors would like to thank the referees for valuable suggestions and remarks. The second author was partially supported by the Center of Excellence for Mathematics and also Research Group on Analysis of Topological and Algebraic Structures of University of Isfahan.

\section{References}

[1] Chen, G. Y., Craven, B. D., Approximate dual and approximate vector variational inequality for multiobjective optimization, J. Austral. Math. Soc. Ser. A 47 (1989), 418-423.

[2] Chen, G. Y., Yang, X. Q., The vector complementary problem and its equivalence with the weak minimal element in ordered space, J. Math. Anal. Appl. 153 (1990), 136-158.

[3] Chiang, Y. Semicontinuous mappings in T.V.S. with applications to mixed vector variational-like inequalities, J. Global Optim. 32 (2005), 467-486.

[4] Daniilidis, A., Hadjisavvas, N., Existence theorems for vector variational inequalities, Bull. Austral. Math. Soc. 54 (1996), 473-481.

[5] Fakhar, M., Zafarani, J., Generalized vector equilibrium problems for pseudomonotone bifunctions, J. Optim. Theory Appl. 126 (2005), 109-124.

[6] Fang, Y. P., Huang, N. J., Existence results for systems of strong implicit vector variational inequalities, Acta Math. Hungar. 103 (2004), 265-279.

[7] Fang, Y. P., Huang, N. J., Strong vector variational inequalities in Banach spaces, Appl. Math. Lett. 19 (2006), 675-680.

[8] Giannessi, F., Theorems of the alternative quadratic programs and complementarity problems, in: "Variational Inequalities and Complementarity Problems" (Proc. Internat. School, Erice, 1978), Wiley, Chichester, 1980, 151-186.

[9] Giannessi, F. (Ed.), Vector Variational Inequalities and Vector Equilibria, Nonconvex Optim. Appl. 38, Kluwer Acad. Publ., Dordrecht, 2000.

[10] Giannessi, F., Maugeri, A. (Eds.), Variational Inequalities and Network Equilibrium Problems (Erice 1994), Plenum, New York, 1995.

[11] Giannessi, F., Maugeri, A. (Eds.), Variational Analysis and Applications, Nonconvex Optim. Appl. 79, Springer, New York, 2005.

[12] Huang, N. J., Li, J., Thompson, H. B., Implicit vector equilibrium problems with applications, Math. Comput. Modelling 37 (2003), 1343-1356.

[13] Huang, N. J., Li, J., On vector implicit variational inequalities and complementarity problems, J. Global Optim. 34 (2006), 399-408.

[14] Konnov, I. V., Yao, J. C., On the generalized vector variational inequality problem, J. Math. Anal. Appl. 206 (1997), 42-58.

[15] Lee, G. M., Kim, D. S., Lee, B. S., Chen, G. Y., Generalized vector variational inequlity and its duality for set-valued maps, Appl. Math. Lett. 11 (1998), 21-26.

[16] Rapcsák, T., On vector complentarity systems and vector variational inequalities, in: "Vector Variational Inequalities and Vector Eequilibria", Nonconvex Optim. Appl. 38, Kluwer Acad. Publ., Dordrecht, 2000.

[17] Schaefer, H. H., Topological Vector Spaces, Springer-Verlag, New York, 1991.

[18] Siddiqi, A. H., Ansari, Q. H., Khaliq, A., On vector variational inequalities, J. Optim. Theory Appl. 84 (1995), 171-180.

[19] Tan, N. X., Tinh, P. N., On the existence of equilibrium points of vector functions, Numer. Funct. Anal. Optim. 19 (1998), 141-156. 
[20] Tian, G. Q., Generalizations of the FKKM theorem and the Ky Fan minimax inequality, with applications to maximal elements, price equilibrium, and complementarity, J. Math. Anal. Appl. 170 (1992), 457-471.

[21] Yang, X. Q., Generalized convex functions and vector variational inequalities, J. Optim. Theory Appl. 79 (1993), 563-580.

[22] Yang, X. Q., On vector variational inequalities: Application to vector equilibria, J. Optim. Theory Appl. 95 (1997), 729-734.

[23] Yang, X. Q., Yu, H., Vector variational inequalities and dynamic traffic equilibria, in: "Variational Analysis and Applications", Nonconvex Optim. Appl. 79, Springer, New York, 2005, 1141-1157.

\author{
J. ZAFARANI \\ DEPARTMENT OF MATHEMATICS \\ UNIVERSITY OF ISFAHAN \\ ISFAHAN 81745-163 \\ IRAN \\ AND \\ SHEIKHBAHAEE UNIVERSITY \\ ISFAHAN, IRAN \\ E-MAIL: JZAF@ZAFARANI.IR
}

T. JABAROOTIAN

Department of MATHEMATiCs

UNIVERSITY OF ISFAHAN

ISFAHAN 81745-163

IRAN

E-MAIL: JABAROOTIAN@YAHOO.COM 